\title{
Mucosa-associated lymphoid tissue lymphoma of the urinary bladder
}

\author{
Taeko Matsuoka $\cdot$ Takayuki Yoshino $\cdot$ Yoshiharu Fukuhara \\ Naoto Miyanaga $\cdot$ Kuniyuki Oka $\cdot$ Reizo Nagayama • \\ Noritoshi Sekido $\cdot$ Katsuya Maebayashi
}

Received: 23 January 2012/ Accepted: 14 May 2012

(C) The Japan Society of Clinical Oncology 2012

\begin{abstract}
A 54-year-old woman presented with hematopyuria. She had experienced difficulty in urinating since undergoing surgery for rectal cancer 15 years earlier. Cystoscopy revealed an edematous mucosa and a submucosal tumor. Computed tomography showed irregular thickening of the bladder wall and tumor-like masses. Although she started clean intermittent self-catheterization to manage the neurogenic bladder, the thickening of the wall became worse. We performed transurethral biopsy of the bladder, and histopathological examination of the specimens revealed non-Hodgkin's lymphoma of the mucosa-associated lymphoid tissue (MALT) type. A diagnosis of stage IE primary MALT lymphoma of the bladder was made. The tumor persisted even after antibiotic therapies for a urinary tract infection and eradication of Helicobacter pylori. Definitive radiotherapy (30 Gy) was administered to the bladder. Subsequent computed
\end{abstract}

T. Matsuoka $(\bowtie) \cdot$ T. Yoshino · Y. Fukuhara $\cdot$ N. Miyanaga Department of Urology, Mito Saiseikai General Hospital,

3-3-10 Futabadai, Mito, Ibaraki 311-4198, Japan

e-mail: taekomatsuoka@gmail.com

K. Oka

Department of Pathology, Mito Saiseikai General Hospital,

Mito, Japan

R. Nagayama

Department of Hematology, Mito Saiseikai General Hospital,

Mito, Japan

N. Sekido

Department of Urology, Institute of Clinical Medicine,

University of Tsukuba, Tsukuba, Japan

K. Maebayashi

Department of Radiation Oncology, Tokyo Women's Medical

University, Tokyo, Japan tomography revealed disappearance of the wall thickening, and transurethral resection showed no residual lesion of lymphoma. She has maintained a complete response for 9 months of follow-up.

Keywords Bladder tumor - Lymphoma · Mucosa-associated lymphoid tissue · Radiotherapy · Neurogenic bladder

\section{Introduction}

Extranodal marginal zone lymphoma of mucosa-associated lymphoid tissue (MALT lymphoma) was first described by Isaacson and Wright [1] in 1983. In the World Health Organization's classification of neoplastic diseases of hematopoietic and lymphoid tissues, MALT lymphoma is defined as an extranodal lymphoma composed of morphologically heterogeneous small B cells including marginal zone (centrocyte-like) cells, monocytoid-like cells, small lymphocytes, and scattered immunoblast and centroblast-like cells [2]. The stomach is the most common site of involvement. MALT lymphoma has also been described in the lung, thyroid, salivary gland, orbit, conjunctiva, and various mucosal sites.

This report describes a case of MALT lymphoma in the urinary bladder of a 54-year-old woman who had chronic inflammation due to neurogenic bladder.

\section{Case report}

A 54-year-old woman with hematopyuria and bladder discomfort was referred to our hospital in March 2010. She had undergone surgery for rectal cancer 15 years earlier, 
and since then she had experienced difficulty in urinating. Although she had visited a local clinic and undergone antibiotic therapy 6 months earlier, the symptoms did not disappear.

Both white blood cells and red blood cells were detected in the urine ( $>100 / \mathrm{HPF})$. Urine culture showed no significant bacterial growth. Cystoscopy showed a flat, sessile, elevated lesion, and urine cytology was negative. Contrastenhanced computed tomography (CT) revealed irregular thickening of the bladder wall and tumor-like masses (Fig. 1).

Initially, because we diagnosed her as having chronic inflammation due to neurogenic bladder, we recommended that she perform clean intermittent self-catheterization to manage the neurogenic bladder. However, CT showed wall thickening progression and right hydronephrosis 3 months after the initial workup (Fig. 2). We therefore performed a transurethral bladder tumor biopsy. Histopathological

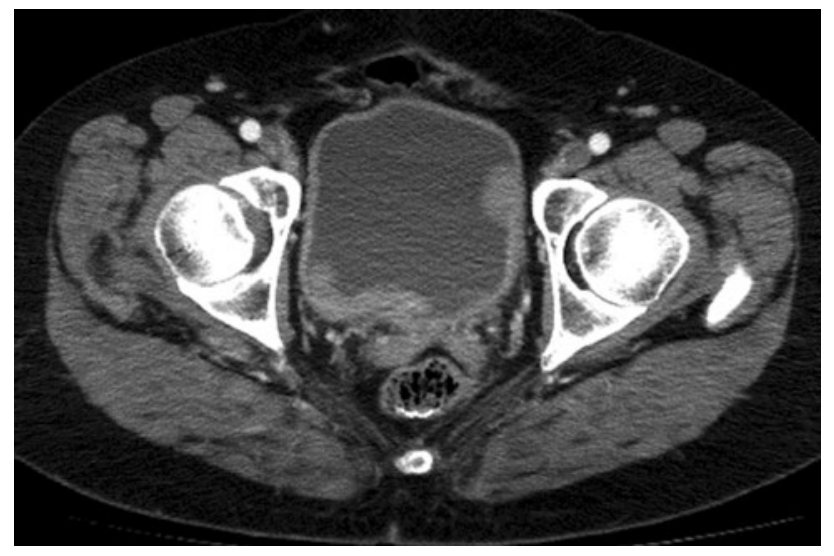

Fig. 1 Contrast-enhanced CT of the bladder at the initial work-up showed thickening of trigone and left bladder wall like submucosal tumors

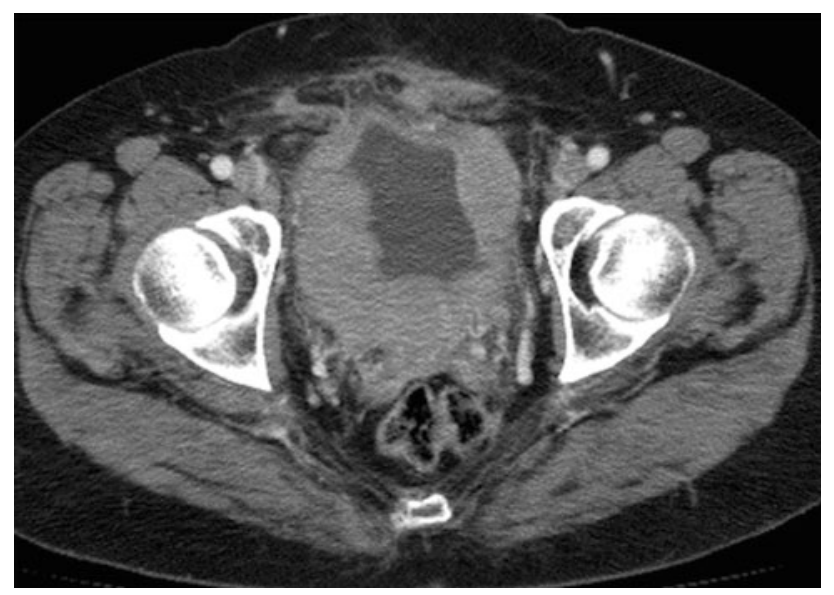

Fig. 2 Contrast-enhanced CT of the bladder before treatment. Thickening of the wall has progressed to the trigone and bilateral bladder wall examination revealed diffuse infiltration of atypical lymphocytes (Fig. 3a), and focal lymphoepithelial lesions (LEL) were also observed (Fig. 3b). Atypical cells were small to medium in size with oval or round nuclei and abundant pale-staining cytoplasms, and resembled monocytoid cells. There was plasma cell differentiation
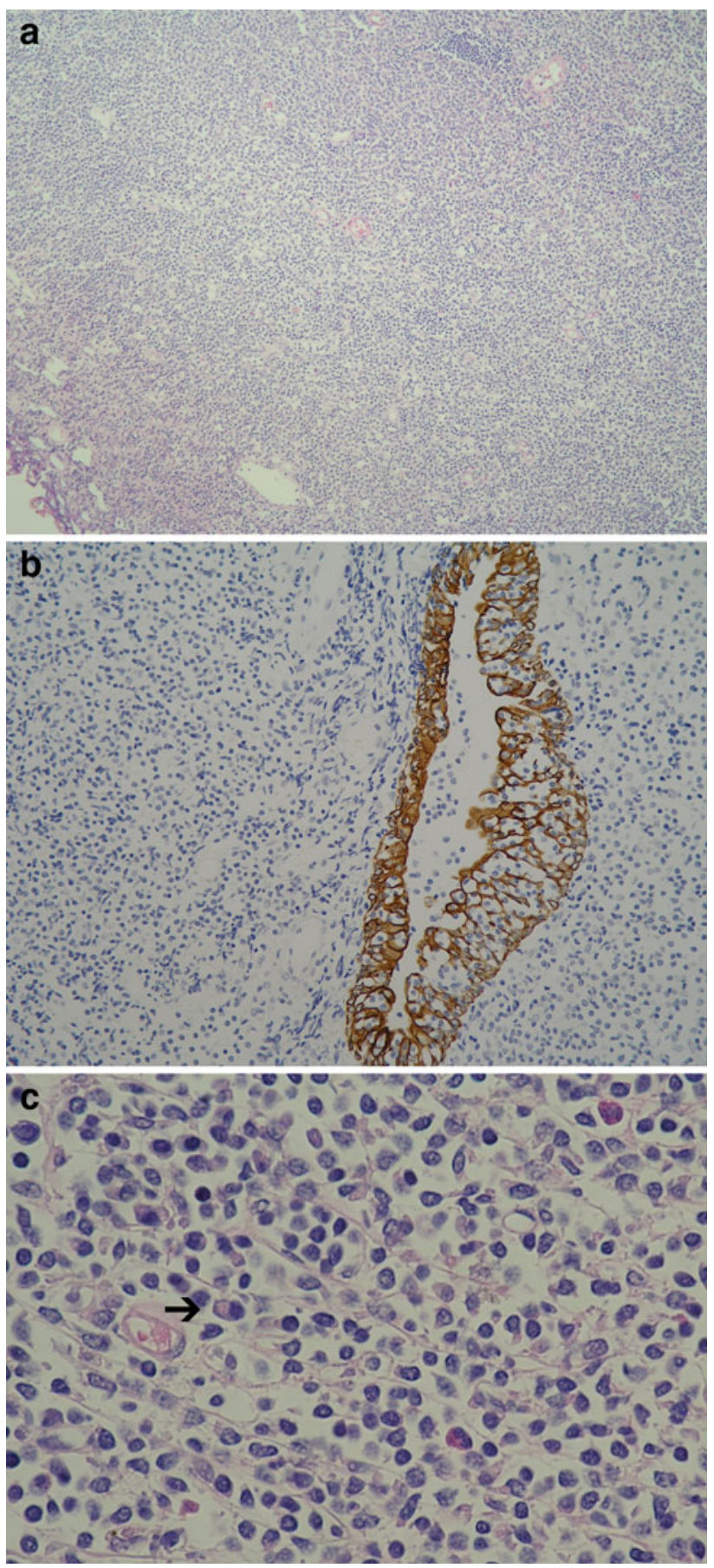

Fig. 3 Histopathological findings. a Diffuse infiltration of atypical lymphocytes (hematoxylin-eosin, $\times 40$ ). b Focal lymphoepithelial lesions in area of Brunn's nest (keratin, $\times 40$ ). $\mathbf{c}$ There was plasma cell differentiation (arrow, hematoxylin-eosin, $\times 400$ ) 

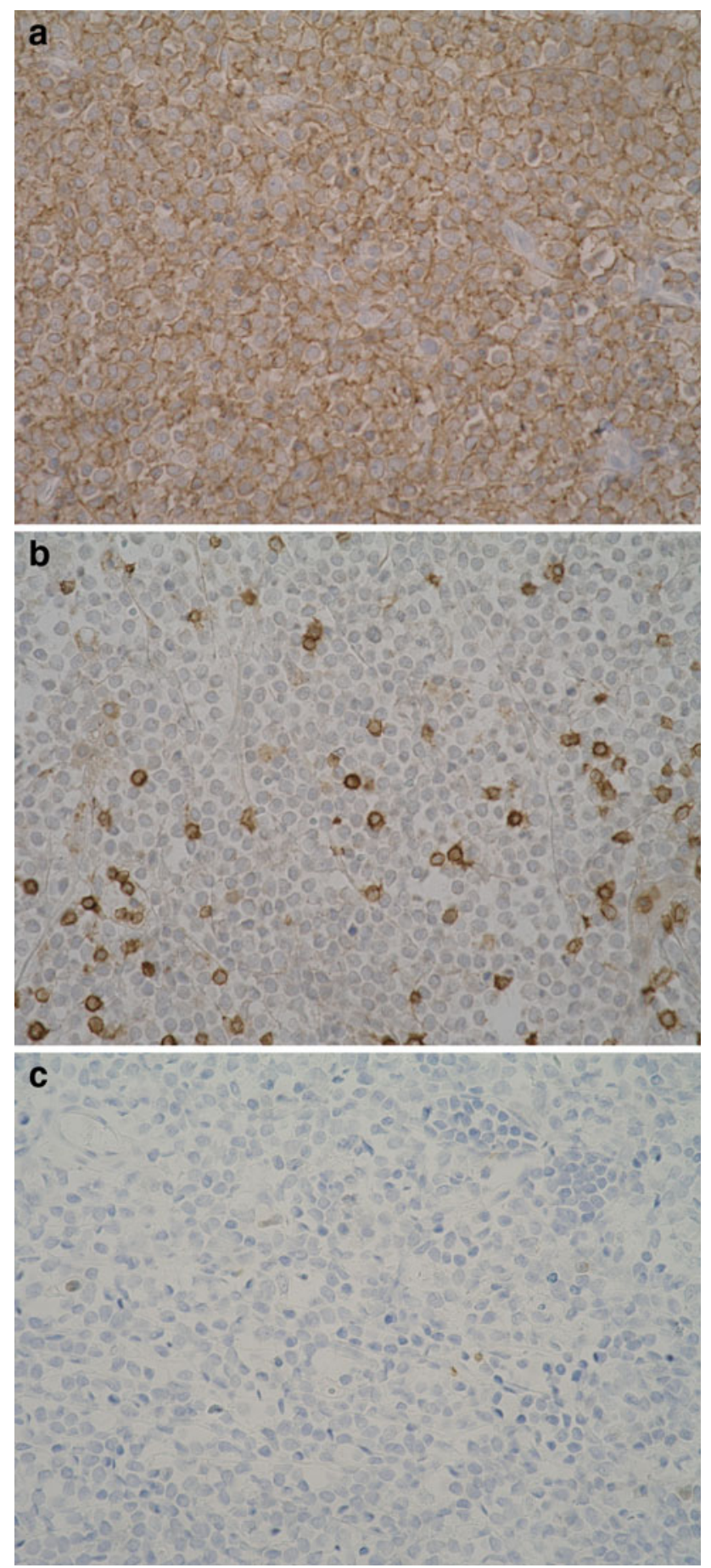

Fig. 4 Atypical cells were positive for CD20 (a), negative for CD3 (b) and CD10 (c). Immunostain, $\times 400$

(Fig. 3c). In almost all fragments obtained by transurethral biopsy, the normal tissue cells had been replaced with lymphoma cells. Immunophenotyping revealed CD20+ (Fig. 4a), CD3 - (Fig. 4b), CD10- (Fig. 4c), CD79a+, bcl-2+, CD5-, cyclin D1- and bcl-6- cells. On the basis of these observations, we diagnosed the bladder tumor as non-Hodgkin's lymphoma of the MALT type.

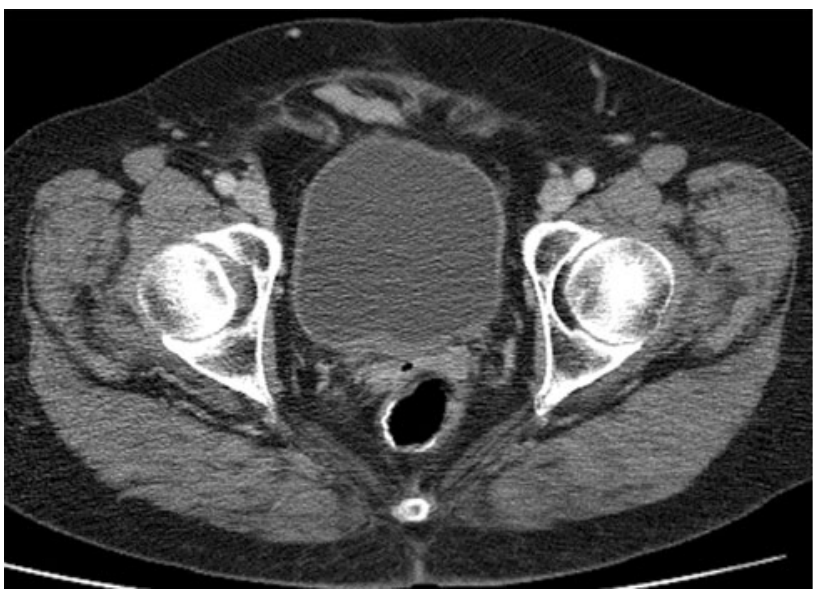

Fig. 5 Post-radiotherapy CT reveals a smooth, flat bladder wall

The results of a CT scan, gallium scintigraphy, positron emission tomography, and bone marrow biopsy suggested that it was a primary MALT lymphoma of the bladder, stage IE (Ann Arbor classification). Although curative radiotherapy was recommended, we were greatly concerned that it might lead to worsening of her bladder function. Urodynamic studies showed acontractile detrusor with low compliance.

Gastric MALT lymphoma is well known to be a slowgrowing disease and related to $H$. pylori infection. There have been reports of extragastric MALT lymphomas responding to anti- $H$. pylori-type antibiotic combinations or antibiotic therapy for urinary tract infection (UTI). Thus, we first treated her with antibiotics, including a cephem antibiotic. We also treated her to eradicate $H$. pylori, although the urea breath test was negative for $H$. pylori. One month later she underwent transurethral bladder tumor biopsy, which showed residual disease. Therefore, definitive radiation therapy ( $30 \mathrm{~Gy}$ ) was administered to the bladder. Subsequent CT revealed disappearance of the wall thickening (Fig. 5), and transurethral resection showed no residual lesion of malignant lymphoma. She has maintained a complete response (CR) for 9 months of follow-up at the time of writing. Follow-up urodynamic studies for evaluation of bladder function are scheduled.

\section{Discussion}

The earliest recorded case of primary lymphoma of the urinary bladder dates back to 1885 , with just over 100 cases now reported [3]. The median age of patients at diagnosis is 69 (range 20-87) years. There is a notable female predominance of $2.7: 1$. Hematuria is the most common presenting symptom, followed by dysuria.

MALT lymphoma is a low-grade extranodal lymphoma whose histogenesis is from MALT [1]. The stomach is the 
most common and best-studied mucosal site of involvement [4]. However, this disease has also been described in various non-gastrointestinal sites, such as the lung, thyroid, salivary gland, conjunctiva, and orbit [5]. Kempton et al. [4] described that MALT lymphoma is the most frequently observed type of primary bladder lymphoma. To our knowledge, 20 cases of MALT lymphoma of the urinary bladder have been reported in Japan [6-11]. Among them, the median age at diagnosis was 69 (range 27-85) years, and there was a female predominance of 9:1.

Many cases of MALT lymphoma have a history of chronic inflammation. H. pylori infection is well known as a cause of gastric MALT lymphoma [5]. Development of MALT lymphoma has also been reported to be associated with autoimmune diseases such as Sjögren's syndrome or Hashimoto's disease. Chromosomal abnormalities are another reported cause [12].

Chronic or recurrent UTI is a significant problem in all patients with neurogenic bladder. Patients with persistent bacteriuria or recurrent UTI have been shown to have significant B cell infiltration into the submucosa, including the lymphoid nodules. These inflammatory changes are likely due to antigenic stimulation from repeated exposure to bacteria [13]. Bacterial colonization of the gastric mucosa with $H$. pylori is known to trigger lymphoid infiltration and the formation of acquired MALT [14]. In our case, cystitis induced by repeated or prolonged UTI with neurogenic bladder might have been associated with the onset of the MALT lymphoma. To the best of our knowledge this is the first report of MALT lymphoma with neurogenic bladder dysfunction.

Patients with localized MALT lymphomas are usually referred for radiation, or sometimes for surgery, chemotherapy, immunotherapy, or a combination of those treatments. Goda et al. [15] reported the long-term outcome of localized MALT lymphomas treated with radiotherapy: the CR rate was $99 \%$, the 10-year recurrence-free rate was $76 \%$, and the overall survival rate was $87 \%$. These findings suggest that radiotherapy is a good choice in the interest of organ preservation, while also producing the desired therapeutic effect. Almost all patients who undergo radiotherapy receive $25-35 \mathrm{~Gy}$ of radiation as curative therapy. In the Japanese series, 13 of 20 patients had radiotherapy as treatment. Seven patients received chemotherapy such as CHOP or THPCOP, and three patients had rituximab alone or in combination with CHOP chemotherapy [6-11].

Eradication of H. pylori is established as an effective initial treatment for stage I gastric MALT lymphoma, and successful eradication leads to $\mathrm{CR}$ of the lymphoma in $80 \%$ of cases [16]. Some cases of extragastric MALT lymphoma have also been treated by eradication of H. pylori. Fujimura et al. [9] reported that the lymphoma disappeared in a case of MALT lymphoma of the bladder with UTI that was treated with antibiotics for 2 weeks and with $H$. pylori eradication therapy. Bosch et al. [17] also reported the disappearance of a MALT lymphoma of the bladder after $H$. pylori eradication monotherapy.

On the other hand, Grünberger et al. [18] reported that H. pylori eradication was ineffective for the treatment of extragastric MALT lymphomas. They suggested that $H$. pylori does not play a role in the development of these lymphomas. The positive rate of $H$. pylori is more than $90 \%$ in gastric MALT lymphomas, but only $45 \%$ in extragastric MALT lymphomas. In our case, residual tumor tissue was observed in the pathological specimen after antibiotic therapy, but subsequent radiation therapy achieved CR. It is clear that this patient will require longterm follow-up of bladder function to evaluate the radiation-related adverse events.

In conclusion, MALT lymphomas can have a favorable prognosis, and patients can receive safe treatment with preservation of their quality of life. We hope to determine the cause of this disease from a molecular biological approach.

Acknowledgments The authors thank Professor Masayuki Noguchi (Department of Pathology, Institute of Basic Medical Sciences, University of Tsukuba) for his kind help and advice.

Conflict of interest The authors declare that they have no conflict of interest.

\section{References}

1. Isaacson P, Wright DH (1983) Malignant lymphoma of mucosaassociated lymphoid tissue. A distinctive type of B-cell lymphoma. Cancer 52:1410-1416

2. Swerdlow SH, Campo E, Harris NL et al (2008) WHO classification of tumours of haematopoietic and lymphoid tissues. IARC, Lyon, pp 214-217

3. Hughes M, Morrison A, Jackson R (2005) Primary bladder lymphoma: management and outcome of 12 patients with a review of the literature. Leuk Lymphoma 46:873-877

4. Kempton CL, Kurtin PJ, Inwards DJ et al (1997) Malignant lymphoma of the bladder: evidence from 36 cases that low-grade lymphoma of the MALT-type is the most common primary bladder lymphoma. Am J Surg Pathol 21:1324-1333

5. Wotherspoon AC, Ortiz-Hidalgo C, Falzon MR et al (1991) Helicobacter pylori-associated gastritis and primary B-cell gastric lymphoma. Lancet 338:1175-1176

6. Hatano K, Sato M, Tsujimoto Y et al (2007) Primary mucosaassociated lymphoid tissue (MALT) lymphoma of the urinary bladder associated with left renal pelvic carcinoma: a case report (in Japanese). Hinyokika Kiyo 53:57-60

7. Kakuta Y, Katoh T, Saitoh J et al (2006) A case of primary mucosa-associated lymphoid tissue lymphoma of the bladder regressed after rituximab in combination with $\mathrm{CHOP}$ chemotherapy (in Japanese). Hinyokika Kiyo 52:951-954

8. Ueno Y, Sakai H, Tsuruta T et al (2007) Mucosa-associated lymphoma of the bladder with relapse in the stomach after successful local treatment. Hinyokika Kiyo 53:575-579 
9. Fujimura M, Chin K, Sekita N et al (2008) Regression of mucosaassociated lymphoid tissue lymphoma of the bladder after antibiotic therapy: a case report (in Japanese). Hinyokika Kiyo 54: 783-786

10. Terasaki Y, Okumura H, Ishiura Y et al (2008) Primary mucosaassociated lymphoid tissue lymphoma of the urinary bladder successfully treated by radiotherapy and rituximab (in Japanese). Rinsho Ketsueki 49:30-34

11. Takei Tomoyuki, Inoue Masaharu, Kasiwagi Bunzou et al (2003) A case of primary mucosa-associated lymphoid tissue (MALT) lymphoma of the urinary bladder diagnosed by TUR biopsy (in Japanese). Rinsho Hinyokika 63:995-999

12. Zucca E, Conconi A, Pedrinis E et al (2003) Nongastric marginal zone B-cell lymphoma of mucosa-associated lymphoid tissue. Blood 101:2489-2495

13. Schlager TA, LeGallo R, Innes D et al (2011) B cell infiltration and lymphonodular hyperplasia in bladder submucosa of patients with persistent bacteriuria and recurrent urinary tract infections. J Urol 186:2359-2364
14. Du MQ, Isaccson PG (2002) Gastric MALT lymphoma: from aetiology to treatment. Lancet Oncol 3:97-104

15. Goda JS, Gospodarowicz M, Pintilie M et al (2010) Long-term outcome in localized extranodal mucosa-associated lymphoid tissue lymphomas treated with radiotherapy. Cancer 116:38153824

16. Wündisch T, Thiede C, Morgner A et al (2005) Long-term follow-up of gastric MALT lymphoma after Helicobacter pylori eradication. J Clin Oncol 23:8018-8024

17. van den Bosch J, Kropman RF, Blok P et al (2002) Disappearance of a mucosa-associated lymphoid tissue (MALT) lymphoma of the urinary bladder after treatment for Helicobacter pylori. Eur J Haematol 68:187-188

18. Grünberger B, Wöhrer S, Streubel B et al (2006) Antibiotic treatment is not effective in patients infected with Helicobacter pylori suffering from extragastric MALT lymphoma. J Clin Oncol 24:1370-1375 